\title{
Numerical Integration of Variational Equations
}

\author{
By James D. Riley, ${ }^{*}$ Morris M. Bennett ${ }^{\dagger}$ and Emily McCormick ${ }^{\dagger}$
}

Introduction. In solving a system of algebraic equations it is well known that the problem is much simpler if the equations are linear. In solving a system of differential equations, one usually does not really care if the equations are linear or not, even though a simplification is possible in the linear case; namely by observing that in a predictor-corrector method the corrector can be solved explicitly for the unknown without predicting. This simplification effectively iterates the corrector to convergence with just a single computation, giving better accuracy with less computing.

It frequently happens, however, that a system of nonlinear equations gives rise to certain linearities. Two such instances, one of considerable importance, are considered here.

The first instance occurs whenever variational equations are solved along with the nonlinear differential equations. Variational equations are always linear. As will be shown, considerable saving in computing and storage is possible while at the same time achieving an increase in accuracy.

The second instance, considered in the Appendix, occurs whenever a higher order equation is reduced to a system of first-order equations. The substitution equations are not only linear but uncoupled. Again an increase in accuracy may be possible with less computing and storage.

Solving Variational Equations. Variational equations may come from various kinds of equations. A somewhat special type will be considered first in order to illustrate the method.

Let the differential equations, nonlinear and hereafter referred to as the primary equations, be as follows:

$$
Y^{\prime \prime}=F\left(x, Y, Y^{\prime}\right),
$$

where $Y$ and $F$ are vectors of the same dimension and where prime refers to differentiation with respect to the independent variable $x$. (Note that the free flight equations of motion are of the preceding form with dimension 3 [1], [2].) The particular solution $Y$ will depend on certain initial conditions and on possibly certain parameters which occur in the function $F$. Let $\beta_{k}(k=1, \cdots, n)$ denote either an initial value or one of the differential equation parameters. The variational equations with respect to $\beta_{k}$ will then be as follows:

$$
Y_{\beta_{k}}^{\prime \prime}=\frac{\partial F}{\partial Y^{\prime}} Y_{\beta_{k}}^{\prime}+\frac{\partial F}{\partial Y} Y_{\beta_{k}}+\frac{\partial F}{\partial \beta_{k}} .
$$

Here $\partial F / \partial Y$ is a matrix whose $i, j$ th component is $\partial F_{i} / \partial Y_{j}$, the partial of the $i$ th component of $F$ with respect to the $j$ th component of $Y$, and similarly for $\partial F / \partial Y^{\prime}$.

Received March 21, 1966. Revised August 3, 1966.

* Hughes Aircraft Company.

$\dagger$ Aerospace Corporation. 
The initial values are determined by differentiating the initial values of $Y$ with respect to $\beta_{k}$ and in general will be zero if $\beta_{k}$ is a differential equation parameter (i.e., a parameter occurring in the function $F$ ). On the other hand, if $\beta_{k}$ denotes an initial value, then not all of the initial conditions on $Y_{\beta_{k}}$ will be zero, but normally $\partial F / \partial \beta_{k}$ will be the null vector. The solution $Y_{\beta_{k}}$ then gives information about the changes in $Y$ if the parameter $\beta_{k}$ is changed.

The coefficient matrices $\partial F / \partial Y$ and $\partial F / \partial Y^{\prime}$ are of course evaluated in terms of corresponding solutions of the primary equations, and likewise the vector $\partial F / \partial \beta_{k}$. The variational equations are solved simultaneously with the nonlinear primary equations and it is often the case that $n$ is quite large. Note however that the solution of the primary equations is independent of the variational equations.

Denote $Y_{\beta}$ by $V\left(\beta=\beta_{1}, \cdots, \beta_{n}\right)$ and $\partial F / \partial Y^{\prime}$ by $A(x)$, also $\partial F / \partial Y$ by $B(x)$ and $\partial F / \partial \beta$ by $C(x)$. Let $V^{\prime}=X$. The system of variational equations may then be written as:

$$
X^{\prime}=A(x) X+B(x) \dot{V}+C(x), \quad V^{\prime}=X .
$$

Assume the integration has been started and another step is to be taken by, for example, the Adams-Moulton fifth order formula [5]. The primary equations may be integrated to the $(n+1)$ st step in the usual manner. Then the implicit corrector formula gives:

$$
\begin{aligned}
X_{n+1}= & X_{n}+\frac{h}{720}\left[251 X_{n+1}^{\prime}+646 X_{n}^{\prime}-264 X_{n-1}^{\prime}+106 X_{n-2}^{\prime}-19 X_{n-3}^{\prime}\right] \\
= & X_{n}+\frac{251 h}{720}\left[A_{n+1} X_{n+1}+B_{n+1} V_{n+1}\right] \\
& +\frac{h}{720}\left[251 C_{n+1}+646 X_{n}^{\prime}-264 X_{n-1}^{\prime}+106 X_{n-2}^{\prime}-19 X_{n-3}^{\prime}\right] \\
= & \frac{251 h}{720}\left[A_{n+1} X_{n+1}+B_{n+1} V_{n+1}\right]+\hat{X},
\end{aligned}
$$

where

$$
\hat{X}=X_{n}+\frac{h}{720}\left[251 C_{n+1}+646 X_{n}^{\prime}-264 X_{n-1}^{\prime}+106 X_{n-2}^{\prime}-19 X_{n-3}^{\prime}\right]
$$

and

$$
\begin{aligned}
V_{n+1} & =V_{n}+\frac{h}{720}\left[251 V_{n+1}^{\prime}+646 V_{n}^{\prime}-264 V_{n-1}^{\prime}+106 V_{n-2}^{\prime}-19 V_{n-3}^{\prime}\right] \\
& =\frac{251 h}{720} X_{n+1}+\hat{V}
\end{aligned}
$$

where

$$
\hat{V}=V_{n}+\frac{h}{720}\left[646 V_{n}^{\prime}-264 V_{n-1}^{\prime}+106 V_{n-2}^{\prime}-19 V_{n-3}^{\prime}\right] .
$$

The quantities $\hat{X}$ and $\hat{V}$ are known because the integration has proceeded to the 
$n$th step. Substituting for $V^{n+1}$ in the first equation and solving for $X^{n+1}$ gives:

$$
X_{n+1}=\left[I-\frac{251 h}{720}\left(A_{n+1}+\frac{251 h}{720} B_{n+1}\right)\right]^{-1}\left(\frac{251 h}{720} B_{n+1} \hat{V}+\hat{X}\right) .
$$

Here $I$ is an identity matrix of appropriate size and the brackets enclose a matrix to be inverted.

Two observations concerning this matrix are of importance. First, the matrix depends only on the solution of the primary equations and not on which parameter is perturbed. Thus the matrix is inverted once each time step; it is of order equal to the dimension of $Y$; and the same matrix is used for each of the parameters $\beta_{i}$. Secondly, as $h$ approaches zero, the matrix approaches the identity matrix, and thus if $h$ is not too large the matrix inversion is well conditioned.

Note also that the storage of $X_{n-4}^{\prime}$ and $V_{n-4}^{\prime}$, which would be required for a fifth order predictor formula for $X^{n+1}$ and $V^{n+1}$ is not required here, thus effecting a saving in storage as well as computation in order to get a more accurate value; more accurate since the (effectively) iterated Adams-Moulton value is obtained.

Variational equations are generally better behaved than the primary equations and usually need not satisfy as stringent accuracy requirements. Thus a further storage savings can be effected by integrating the variational equations with lower order formulas than the primary equations.

The preceding scheme, using the fifth order Adams-Moulton formulas, has been employed in an orbit determination program wherein the primary equations are the second order nonlinear differential equations of motion [1], [2] $\ddot{X}=-\mu X / r^{3}+F$ where:

$\mu=$ gravitational constant (GM) of the earth,

$r=|X|=\left(x^{2}+y^{2}+z^{2}\right)^{1 / 2}$,

$F=F_{1}+F_{2}+F_{3}+F_{4}=$ perturbative acceleration due to asphericity of the earth, extraterrestrial gravitational forces, atmospheric drag and low thrust respectively.

The variational equations for 60 parameters have been integrated simultaneously with the motion equations and since each parameter gives rise to 3 differential equations (one for each component of satellite position) the total system consisted of 183 differential equations. The motion equations ( 3 in number) were integrated with a sixth order Gauss-Jackson package which requires 25 storage cells per equation. The remaining variational equations (180 in number) were integrated as described previously and required only 12 storage cells per equation. This configuration has resulted in accuracies comparable to those achieved when the entire 183 equations were integrated with the Gauss-Jackson package $\ddagger$ and has noticeably reduced the machine time required for such integrations.

$\ddagger$ So called "delta tests" were used to test the accuracy. These tests consisted of the following steps:

(1) integrating a reference trajectory and the variational equations (in both modes, i.e., sixth order Gauss-Jackson and the formulas developed for fifth order Adams-Moulton) for two days (approximately 2900 steps);

(2) perturbing, one at a time, the parameters for which variational equations were integrated in (1) and integrating a trajectory for the same period of time;

(3) differencing the trajectories obtained in (1) and (2) to determine the prediction accuracy of the partial derivatives generated in (1). 
The stability of this procedure is that of the iterated Adams-Moulton method (see [4]).

A More General System of Variational Equations. The solution of variational equations when the equations of motion include first order as well as second order equations will now be considered.

Let the primary equations be:

$$
\begin{aligned}
Y^{\prime \prime} & =F\left(x, X, Y, Y^{\prime}\right), \\
X^{\prime} & =G(x, X, Y),
\end{aligned}
$$

where $Y$ is a $j$-vector and $X$ is a $k$-vector. The function $G$ could be allowed to depend also on $Y^{\prime}$, but for simplicity this is not considered. (Note the equations of motion for powered flight are of the above form [3] with $j=k=3$.) Let

$$
\frac{\partial F}{\partial Y}=A, \quad \frac{\partial F}{\partial Y^{\prime}}=B, \quad \frac{\partial F}{\partial X}=C, \quad \frac{\partial G}{\partial X}=D, \quad \frac{\partial G}{\partial Y}=E .
$$

$A, B, C, D$, and $E$ are matrices with orders: $A, j \times j ; B, j \times j ; C, j \times k ; D, k \times k$; $E, k \times j$. The variational equations are:

$$
\begin{aligned}
Y_{\beta}^{\prime \prime} & =A Y_{\beta}+B Y_{\beta}{ }^{\prime}+C X_{\beta}+\frac{\partial F}{\partial \beta}, \\
X_{\beta}^{\prime} & =D X_{\beta}+E Y_{\beta}+\frac{\partial G}{\partial \beta} .
\end{aligned}
$$

Let

$$
Y_{\beta}=\bar{Y}, \quad X_{\beta}=\bar{X}, \quad Y_{\beta}^{\prime}=\bar{U} .
$$

Then, proceeding as before, using the fourth order Adams-Moulton corrector formula:

$$
\begin{aligned}
\bar{U}_{n+1}= & \left\{I_{j}-\frac{3 h}{8} B-\left(\frac{3 h}{8}\right)^{2} A-\left(\frac{3 h}{8}\right)^{3} C\left(I_{k}-\frac{3 h}{8} D\right)^{-1} E\right\}^{-1} \\
& \times\left\{R_{n}+\frac{3 h}{8}\left[A P_{n}+C\left(I_{k}-\frac{3 h}{8} D\right)^{-1}\left(Q_{n}+\frac{3 h}{8} E P_{n}\right)\right]\right\}, \\
\bar{Y}_{n+1}= & \frac{3 h}{8} \bar{U}_{n+1}+P_{n}, \\
\bar{X}_{n+1}= & \left(I_{k}-\frac{3 h}{8} D\right)^{-1}\left(\frac{3 h}{8} E \bar{Y}_{n+1}+Q_{n}\right),
\end{aligned}
$$

where

$$
\begin{aligned}
P_{n} & =\bar{Y}_{n}+\frac{h}{24}\left(19 \bar{U}_{n}-5 \bar{U}_{n-1}+\bar{U}_{n-2},\right. \\
R_{n} & =\bar{U}_{n}+\frac{h}{24}\left(19 \bar{U}_{n}^{\prime}-5 \bar{U}_{n-1}^{\prime}+\bar{U}_{n-2}^{\prime}+9 \frac{\partial F}{\partial \beta}\right), \\
Q_{n} & =\bar{X}_{n}+\frac{h}{24}\left(19 \bar{X}_{n}^{\prime}-5 \bar{X}_{n-1}^{\prime}+\bar{X}_{n-2}^{\prime}+9 \frac{\partial G}{\partial \beta}\right),
\end{aligned}
$$


and the matrices $A, B, C, D, E$, are evaluated using the solution of the primary equations at the $(n+1)$ st time step. $I_{j}$ is the $j$ th order identity, and $I_{k}$ is the $k$ th order identity. Note that two matrix inversions are involved at each time step. However, again the inversions are the same for all values of the parameter $\beta$, and again each is well conditioned if the step size $h$ is not too large.

Conclusion. It has been shown in this paper that variational equations may be solved in a much more practical way by taking advantage of their linear nature.

Appendix-Solving Second Order Equations. Let the equations to be solved be of the form:

$$
\begin{aligned}
& y^{\prime \prime}=f\left(x, y, y^{\prime}, w\right), \\
& w^{\prime}=g\left(x, y, y^{\prime}, w\right),
\end{aligned}
$$

where $y$ and $f$ are vectors of order $p$, and $w$ and $g$ are vectors of order $q$, and the prime denotes differentiation with respect to the independent variable $x$. To use the Adams-Moulton procedure the system is reduced to a first order system by letting $y^{\prime}=v$. Thus:

$$
\begin{aligned}
v^{\prime} & =f(x, y, v, w), \\
y^{\prime} & =v, \\
w^{\prime} & =g(x, y, v, w) .
\end{aligned}
$$

The usual procedure is to compute predicted values of $v_{n+1}, y_{n+1}$, and $w_{n+1}$, then use these predicted values to evaluate $v_{n+1}^{\prime}, y_{n+1}^{\prime}$, and $w_{n+1}^{\prime}$. Then corrected values of $v_{n+1}, y_{n+1}$, and $w_{n+1}$ are computed and these are used to re-evaluate $v_{n+1}^{\prime}, y_{n+1}^{\prime}$, and $w_{n+1}^{\prime}$. Note however that as soon as $v_{n+1}$ is predicted, the first evaluation of $y_{n+1}^{\prime}$ is already accomplished. This permits the immediate evaluation of the corrected value of $y_{n+1}$, skipping the evaluation of the predicted value. This corrected value of $y_{n+1}$ can then be used in the first of the two derivative evaluations instead of the bypassed predicted value. Since the corrected value is more accurate than the predicted value this would normally lead to a more accurate derivative evaluation with less computing and less storage of data. The saving in storage of data occurs because $y_{n-k+1}^{\prime}$, where $k$ is the order of the method, used in computing the predicted value of $y_{n+1}$ is not needed in computing the corrected value.

These observations could be generalized to higher order equations. For example, if $z^{\prime \prime \prime}$ is given, the first computed value of $z_{n+1}$ could be what is ordinarily the second corrected value.

The proposed scheme has been employed in a program using fourth order AdamsMoulton formulas. In particular it has been tested on single equations of the form

$$
y^{\prime \prime}=f\left(x, y, y^{\prime}\right) \text {. }
$$

The accuracy obtained is comparable to that of the standard procedure. In some cases it is possible to see that a slight loss in accuracy will occur. For example, the equation $y^{\prime \prime}=\frac{1}{2}\left(y+y^{\prime}\right)$ has as solution $y=e^{x}$. The truncation error is then such that the predicted values will be too small and the corrected values too large. Using the predicted value to evaluate $y_{n+1}^{\prime \prime}$ will make that value too small, but this 
will tend to cancel the truncation error in the corrected value of $y_{n+1}^{\prime}$. The proposed scheme will not benefit from this cancellation.

Note in particular that if the equation is of the form $y^{\prime \prime}=f\left(x, y^{\prime}\right)$, then identical results in the two schemes will be achieved. In any case, savings in storage of data and computation do occur.

The idea expressed in the Appendix, if not the details, has been noted before (see $[6])$.

Aerospace Corporation

Los Angeles, California 90045

1. S. D. Conte, "The computation of satellite orbit trajectories," Advances in Computers, Vol. 3, Academic Press, New York, 1962. MR 28, *2906.

2. R. J. Mercer, et al, Trace, Aerospace Orbit Determination Program, Report No. TDR269 (4110-04)-1, Aerospace Corp., Nov. 1964 (AD 454 404).

3. C. S. Christensen, A. R. JAcobsen \& R. J. Mercer, Trace-C Powered Flight Trajectory Determination Program, Report No. TOR-469(5352)-1, Aerospace Corp., May 1965.

4. R. R. Brown, J. D. Riley \& M. M. BenNetT, "Stability properties of Adams-Moulton type methods," Math. Comp., v. 19, 1965, pp. 90-96. MR 31 *2829.

5. P. Henrici, Discrete Variable Methods in Ordinary Differential Equations, Wiley, New York, 1962. MR 24 *B1772.

6. W. E. MILNe, Numerical Solution of Differential Equations, Wiley, New York; Chapman \& Hall, London, 1953, p. 82. MR 16, 864 . 